\title{
Comportement mécanique des sols lœssiques naturels sous sollicitations cycliques
}

\section{Y.-J. CUI \\ J. CANOU}

École des Ponts ParisTech

UR Navier/CERMES

6 et 8 , avenue Blaise-Pascal

Cité Descartes

Champs-sur-Marne

77455 Marne-la-Vallée

Cedex

cui@cermes.enpc.fr

canou@cermes.enpc.fr

\section{J.-M. TERPEREAU}

SNCF

Direction de l'ingénierie

6 , avenue

François-Mitterrand

93574 La Plaine Saint-Denis

serge.nebieridze@sncf.fr

jean-marc.terpereau@sncf.fr

J.-P. KARAM

Bonnard and Gardel

Consulting Engineers

Lausanne (Switzerland)

jean-paul.karam@bg-21.com
Dans le cadre du plan d'action lancé en 2002 par le Réseau ferré de France (RFF) et la Société nationale des chemins de fer (SNCF), et suite aux fontis observés sur la ligne à grande vitesse Nord (LGV), une étude de laboratoire a été menée visant à identifier les mécanismes d'instabilité liés au comportement du sol concerné : le lœess. Les échantillons de sols intacts ont été prélevés en bloc à différentes profondeurs, et différents essais ont été réalisés : l'essai d'identification géotechnique, l'essai d'effondrement à l'œdomètre, l'essai de fatigue à faible et grande fréquences, l'essai de liquéfaction. Les résultats ont montré un effondrement modéré à partir de l'état naturel $(<3,7 \%)$. En revanche, une grande sensibilité à la teneur en eau a été observée puisqu'une augmentation linéaire de l'effondrement avec la diminution de la teneur en eau a été identifiée. Les résultats des essais de fatigue à faible fréquence ont montré que les déformations augmentent généralement avec l'augmentation de l'amplitude du déviateur. L'effet de fréquence s'est avéré négligeable. Le caractère liquéfiable des sols a été démontré aussi bien par les essais de cisaillement non drainés sous chargements monotones que par les essais cycliques. De plus, les résultats ont montré qu'on peut définir un critère de liquéfaction soit en termes de surpression interstitielle soit en terme de déformation axiale. La résistance à la liquéfaction des sols semble fortement dépendante de la densité et de la fraction des fines.

Mots-clés : lœss naturel, effondrement, fatigue, liquéfaction, fréquence, teneur en eau, sollicitations cycliques.

\section{Mechanical behaviour of natural loessic soils under cyclic loading}

Within the action plan launched in 2002 by the French company for railway management (RFF) and the railway company (SNCF) and following on the instability problem observed on the northern high speed line, a laboratory study was undertaken aiming at identifying the mechanisms of instability related to the behaviour of the soil involved: loess. Intact samples were taken in block at various depths and different tests were carried out: geotechnical classification tests, collapse test in oedometer, fatigue tests at low and high frequencies, and liquefaction tests. The results showed a moderate collapse starting from the natural state $(<3.7 \%)$. On the other hand, a high sensitivity to the water content was observed since a linear increase of collapse with the reduction of the water content was identified. The results of the fatigue tests at low frequency showed that on the whole the strains increase with the increase in amplitude of the deviator stress. The effect of frequency was found to be negligible. The liquefiable character of the soils was shown by both undrained monotonous shear tests and cyclic shear tests. Moreover, the results showed that it is possible to define a criterion of liquefaction either in terms of over pore pressure build-up or in term of axial strain. The liquefaction resistance of the soils seems strongly dependent on the density and the fraction of fines.

Key words: natural loess, collapse, fatigue, liquefaction, frequency, water content, cyclic loadings. 
1

\section{Introduction}

Le nombre de cycles de chargement auquel sont soumises les voies de la ligne à grande vitesse (LGV) Nord est estimé à 3500 par jour, soit 1270000 par an. Au cours de l'hiver 2000-2001 marqué par une pluviométrie exceptionnelle qui a provoqué des inondations de la vallée de la Somme, on a observé de nombreux effondrements en surface du sol, dénommés fontis. Ces fontis observés étaient de tailles variables, les plus importants atteignant environ $5 \mathrm{~m}$ de diamètre. L'origine est attribuée soit aux cavités d'origine militaire (tranchées, sapes, etc.) créées pendant la première guerre mondiale soit à des carrières (extraction de la craie). Le risque d'origine karstique, quoique identifié, s'avère marginal. L'expertise menée par Rat (conseil général des Ponts) pour le compte de la direction des transports terrestres a conduit la SNCF à mener un projet de recherches dédié à la compréhension du mécanisme de formation de ces fontis, à la part relative prise par les conditions hydriques du sol et par les vibrations générées par les circulations ferroviaires. En 2003, des simulations numériques par la méthode des éléments finis ont été effectuées par une entreprise afin d'évaluer les contraintes cycliques dans le sol. Les résultats de calcul ont montré qu'à une distance de $5 \mathrm{~m}$ des voies, l'amplitude du chargement cyclique vertical varie de 11 à $23 \mathrm{kPa}$; et ceci jusqu'à une profondeur de $4 \mathrm{~m}$ et avec un spectre de fréquence autour de $20 \mathrm{~Hz}$. La contrainte diminue considérablement au-delà de $5 \mathrm{~m}$ de profondeur.

Du point de vue mécanique, les fontis observés pourraient être liés au mécanisme d'effondrement avec l'augmentation de la teneur en eau et au mécanisme de fatigue avec les chargements à grands nombres de cycles. On note aussi le possible mécanisme de liquéfaction lorsque le sol est saturé.

Le phénomène d'effondrement lié à l'augmentation de la teneur en eau sous des contraintes mécaniques a fait l'objet de nombreuses investigations, en particulier dans les pays où les sols lœessiques sont répandus (voir Cui et Magnan, 2000). Il est maintenant bien connu que l'effondrabilité d'un sol dépend de sa minéralogie, sa fraction argileuse, la forme de ses grains et sa granulométrie, sa teneur en eau naturelle, son indice des vides, la dimension et la forme de ses pores, son niveau de cimentation, la nature et la concentration des ions dans son eau interstitielle. Sur le plan expérimental, l'effondrement du sol est souvent déterminé à l'œdomètre avec des méthodes connues sous les noms du simple œdomètre et du double œdomètre (Jennings et Knight, 1957 ; Knight, 1963). L'avantage du simple œdomètre est qu'on détermine l'effondrement du sol en suivant le chemin réel, alors que l'avantage du double œedomètre est qu'on balaye avec deux essais toute la gamme de contraintes. Contrairement au cas statique, l'effet cyclique sur l'effondrement est très peu abordé, tant sur le plan théorique qu'expérimental. Cui et al. (1995) ont montré que cet effet dynamique est loin d'être négligeable. En effet, pour un sol lœessique sous $200 \mathrm{kPa}$ de contrainte à l'œdomètre, ils n'ont obtenu qu'environ $2 \%$ d'effondrement sous l'effet de la saturation, alors qu'avec vibration, l'effondrement a atteint $10 \%$

Sous l'effet de grands nombres de cycles de chargement cyclique, le sol présente le comportement de fatigue. Ce comportement est fortement conditionné par l'état hydrique du sol. Quand le sol atteint l'état saturé, le chargement cyclique en condition non drainée pourrait donner lieu au phénomène de liquéfaction. De plus, pour ces comportements de fatigue et de liquéfaction, la fréquence pourrait jouer un rôle important.

Dans le présent travail, on étudie expérimentalement le comportement des loess prélevés en bloc à différentes profondeurs sur un site proche des voies de la LGV Nord. On a d'abord analysé les sols à partir d'essais d'identification géotechniques ; puis on a réalisé différents types d'essais mécaniques pour caractériser le comportement d'effondrement, de fatigue et de liquéfaction de ces sols.

\section{2 \\ Matériaux}

Les sols étudiés ont été prélevés dans le nord de la France, à environ $140 \mathrm{~km}$ de Paris. Le prélèvement a été effectué selon la norme française (AFNOR, 1994) : une tranchée de $1,5 \times 9 \mathrm{~m}$ a été ouverte, à une distance de $25 \mathrm{~m}$ de la plate-forme ferroviaire. Quand on a atteint $1,2 \mathrm{~m}$ de profondeur, un premier bloc témoin a été découpé à l'aide de spatules. Le bloc a été ensuite placé dans une boite en plasticue dont la face ouverte a été recouverte par un film plastique, puis scellée avec de la paraffine. La même opération a été répétée pour d'autres profondeurs : $2,2 \mathrm{~m}, 3,5 \mathrm{~m}$ et $4,9 \mathrm{~m}$. La figure 1 montre que les courbes granulométriques des quatre sols sont comparables et typiques du lœss, avec une fraction argileuse comprise entre 16 et $20 \%$. Les sols à $1,2 \mathrm{~m}$ et à $4,9 \mathrm{~m}$ présentent une fraction argileuse légèrement plus grande (18 et $20 \%$ respectivement) que les deux autres $(16 \%)$. Les propriétés géotechniques des sols sont présentées dans le tableau I. La limite de liquidité $\left(\mathrm{w}_{\mathrm{L}}\right)$ varie de 26 à $30 \%$; l'indice de plasticité (I) varie de 6 à 9 ; la densité sèche $\left(\rho_{\mathrm{d}}\right)$ est plutôt petite $\left(1,39-1,55 \mathrm{Mg} / \mathrm{m}^{3}\right)$ correspondant à une porosité élevée $(n=0,43-0,49)$ ou à un grand indice des vides $(e=0,76-0,93)$; la teneur en carbonate est assez élevée (5 - $15 \%$ ). La diffraction aux rayons $\mathrm{X}$ a montré que la fraction argileuse est constituée de kaolinite, d'illite et d'illite-smectite interstratifiées. La succion mesurée en utilisant la méthode du papier filtre (voir le détail dans Delage et Cui, 2000) n'est pas élevée, allant de 13,8 à 34,1 kPa, bien que le degré de saturation $\left(\mathrm{S}_{\mathrm{r}}\right)$ soit assez bas, compris entre 53 et $82 \%$ (teneur en eau naturelle initiale $\mathrm{w}_{\mathrm{i}}=16,6-23,7 \%$ ).

TAbtequr Propriétés géotechniques des sols étudiés. Geotechnical properties of the soils studied.

\begin{tabular}{|c|c|c|c|c|c|c|c|c|c|c|}
\hline $\begin{array}{l}\text { Profurdevir } \\
\text { (m) }\end{array}$ & $\begin{array}{l}0.85 \\
2 \min \end{array}$ & $(6.6)$ & 1 & Matin) & n & 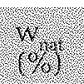 & e & $\begin{array}{l}5 \\
(5)\end{array}$ & $\begin{array}{l}\mathrm{Carg}) \\
\mathrm{fer}\end{array}$ & $\begin{array}{l}\text { Succon } \\
\text { (kpa) }\end{array}$ \\
\hline 1,2 & 20 & 30 & 9 & 1,52 & 0,44 & 18,9 & 0,76 & 66 & 5 & 20 \\
\hline 2,2 & 16 & 28 & 9 & 1,39 & 0,49 & 18,1 & 0,93 & 53 & 6 & 34 \\
\hline 3,5 & 16 & 26 & 6 & 1,54 & 0,43 & 16,6 & 0,82 & 55 & 15 & 27 \\
\hline 4,9 & 18 & 30 & 9 & 1,55 & 0,43 & 23,7 & 0,78 & 82 & 9 & 14 \\
\hline
\end{tabular}




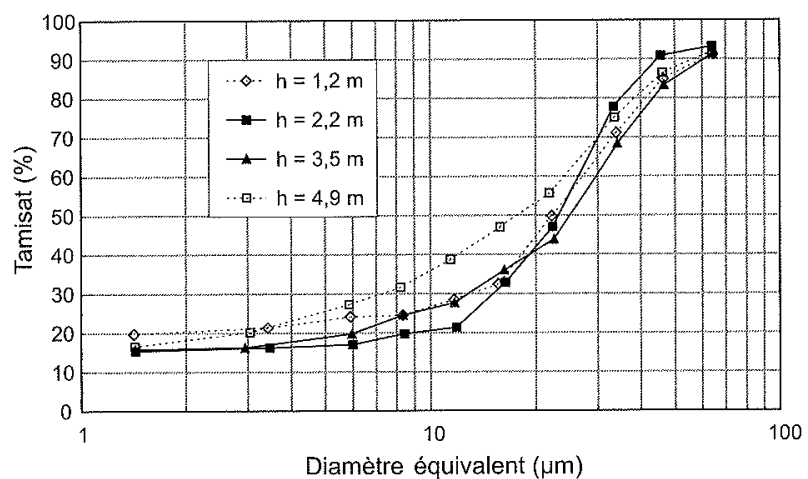

no.1 Courbes granulométriques des quatre sols étudiés.

Grain size distribution curves of the four soils studied.
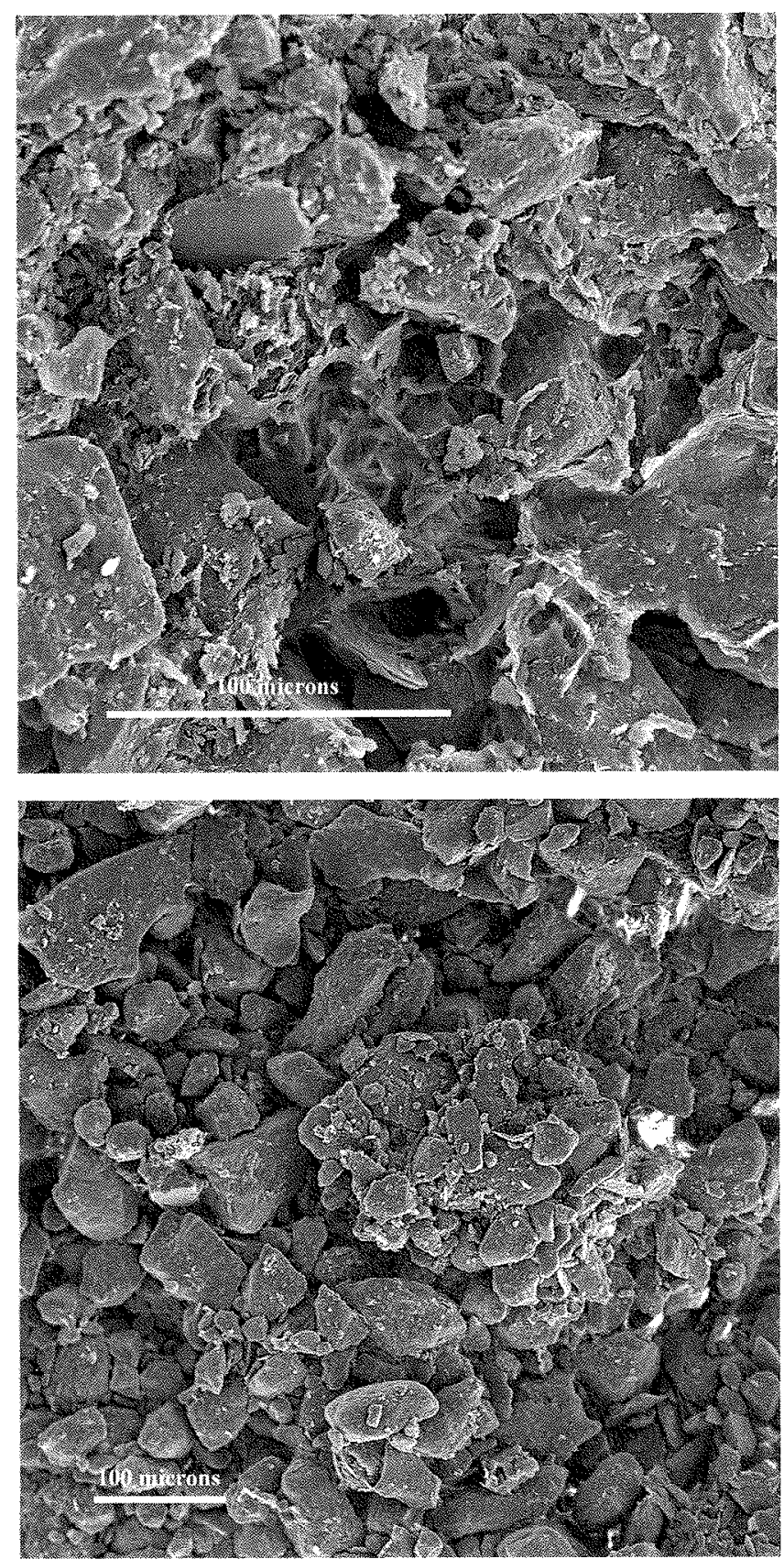

La figure 2 montre la microstructure des quatre sols, observée au microscope électronique à balayage (MEB). Le sol à 1,2 $\mathrm{m}$ de profondeur (Fig. 2a) présente une microstructure assez compacte avec peu de grands pores visibles; la fraction argileuse parait omniprésente. Le sol à 2,2 m présente en revanche des pores assez grands (Fig. 2b) ; les grains de silt sont collés par les fines argileuses pour former ensemble des agrégats. La figure 2c présente la microstructure du sol à $3,5 \mathrm{~m}$ de profondeur. On y identifie peu d'argile et les grains de silt semblent bien séparés. La plupart des grains mesurent entre 20 et $40 \mu \mathrm{m}$, ce qui est compatible avec la courbe granulométrique (Fig. 1) ; les pores entre grains sont petits et mesurent quelques micromètres. Le sol à 4,9 m (Fig. 2d) présente une microstructure assez argileuse avec des grands pores allant jusqu'à quelques micromètres. Cette observation est en bon accord
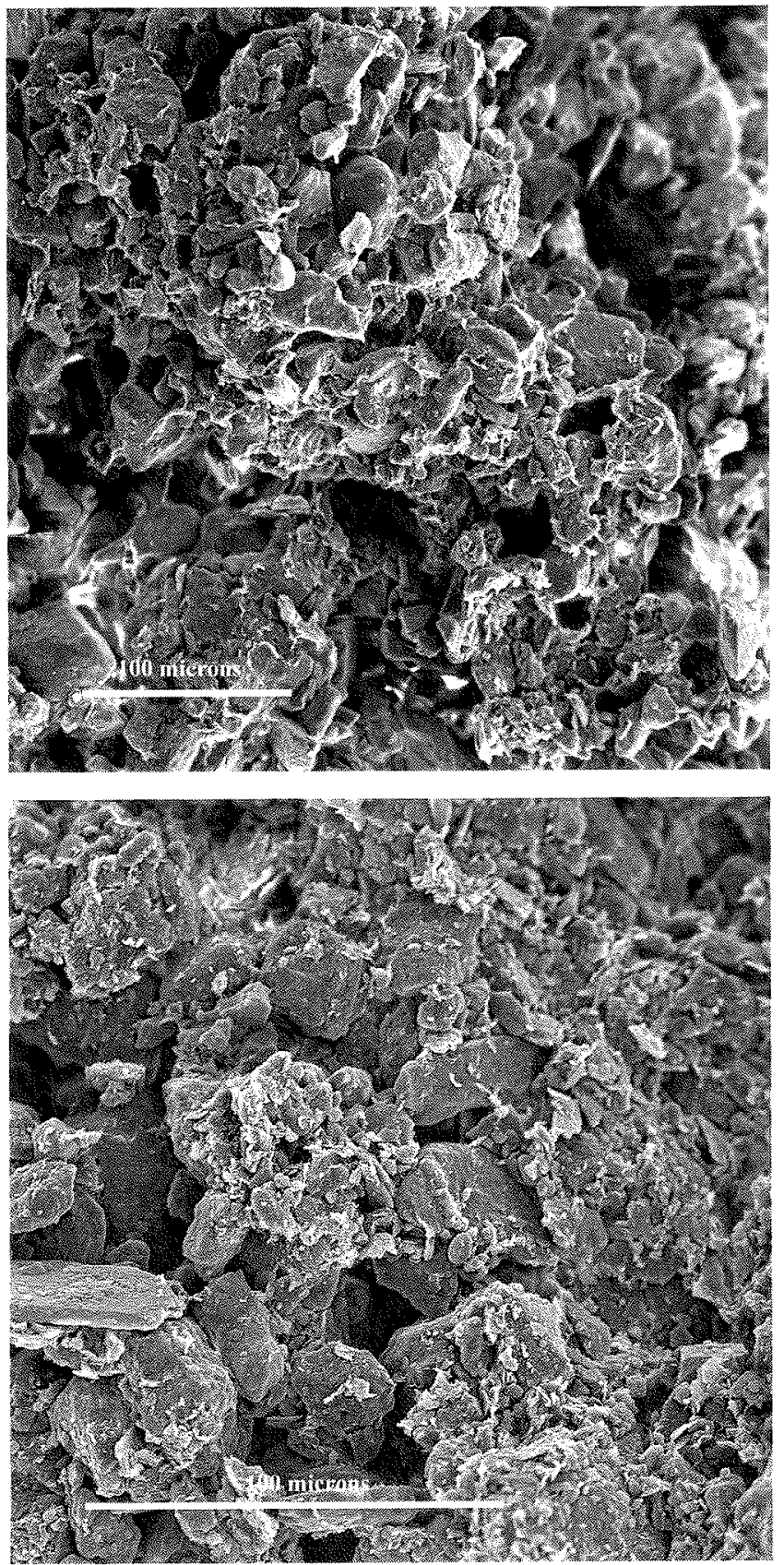

16. 2 Microstructure des sols observée au microscope électronique au balayage. (a) sol à 1,2 m ; (b) sol à 2,2 m ; (c) sol à $3,5 \mathrm{~m}$; (d) sol à $4,9 \mathrm{~m}$.

Microstructure of soils observed at scanning electron microscope. (a) soil at $1.2 \mathrm{~m}$; (b) soil at $2.2 \mathrm{~m}$; (c) soil at $3.5 \mathrm{~m}$; (d) soil at $4.9 \mathrm{~m}$. 


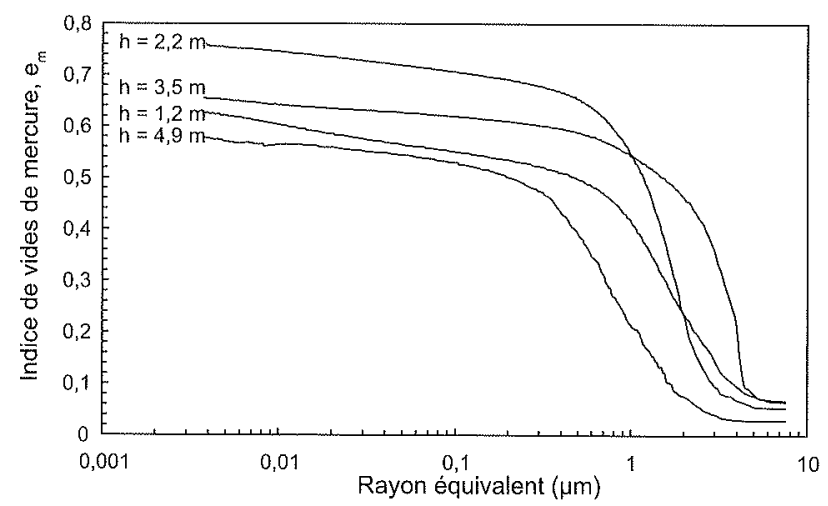

16. 3 Courbes de distribution des pores des sols étudiés $\left(e_{m}\right.$ est le rapport du volume de mercure sur le volume des solides).

Pore size distribution curves of the soils studied $\left(e_{m}\right.$ is the ratio of mercury volume to solids volume).

avec les courbes porosimétriques obtenues en utilisant la méthode de porosimétrie à mercure sur des échantillons lyophilisés (Fig. 3). En effet, on observe que pour les quatre sols, les familles de pores se trouvent entre 0,8 et $4 \mu \mathrm{m}$. Les valeurs d'indice des vides identifiées montrent bien que le sol à 2,2 m est le plus lâche, viennent ensuite les sols à $3,5 \mathrm{~m}$, à $1,2 \mathrm{~m}$ et à $4,9 \mathrm{~m}$ de profondeur, ce qui est assez compatible avec les indices des vides déterminés de façon globale sur des échantillons plus grands. On note cependant une valeur systématiquement moins grande obtenue pour les quatre sols par la méthode porosimétrique, à relier à de très gros pores non identifiables par un porosimètre standard qui opère de la pression atmosphérique jusqu'à $2 \mathrm{MPa}(\mathrm{Osipov}$ et Sokolov, 1995; Cui et al., 1998).

\section{3}

\section{Méthodes expérimentales}

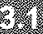

\section{Effondrement à l'œdomètre}

Pour évaluer l'effondrabilité des sols, on a réalisé une série d'essais au double et au simple œdomètre. Les échantillons ont été préparés par carottage à partir de petits blocs de sol (environ $50 \times 100 \times 100 \mathrm{~mm}$ ) et avaient une dimension de $20 \mathrm{~mm}$ de hauteur et de $70 \mathrm{~mm}$ de diamètre. L'essai au double cedomètre consiste à réaliser deux courbes de compression, une à teneur en eau constante et l'autre sur un échantillon initialement saturé sous une faible charge de $3 \mathrm{kPa}$. Ainsi, pour toute la gamme de contraintes, la différence de déformations entre les deux courbes indique le degré d'effondrement. L'essai au simple odomètre consiste à déterminer une courbe de compression unique, commençant à teneur en eau constante jusqu'à une contrainte de $200 \mathrm{kPa}$ sous laquelle on sature l'échantillon pour observer l'effondrement.

L'effondrabilité des quatre sols prélevés a été déterminée en utilisant les deux méthodes et à partir d'un état initial correspondant à la teneur en eau initiale. L'effet de la teneur en eau initiale a été étudié spécificuement sur le sol à 2,2 $\mathrm{m}$ de profondeur. Pour ce faire, les blocs de sol ont été soit humidifiés, soit séchés pour atteindre la teneur en eau voulue $(23,18,14,10$, 4 et $0 \%$ ) avant les carottages.

\section{9}

\section{Fatigue au triaxial à faible fréquence}

Les essais de fatigue en condition triaxiale ont été réalisés en utilisant une presse triaxiale cyclique équipée avec un système de suivi de volume développé par Cui et al. (2007). Pour la préparation des échantillons, des blocs de sol d'environ $150 \times 150 \times 300 \mathrm{~mm}$ ont été réalisés. Ils ont été ensuite amenés à la teneur en eau voulue $(10,14,21,5,23$ et $27 \%$ ) soit par séchage soit par humidification. On a ensuite réalisé des échantillons de $70 \mathrm{~mm}$ de diamètre et $140 \mathrm{~mm}$ de hauteur par taille.

Étant donné que le système de suivi de volume ne permet pas des essais à fréquence élevée, on a adopté une fréquence de $0,05 \mathrm{~Hz}$. Ce choix est aussi justifié par les observations de Canou et al. (2002) qui ont montré que dans la gamme de faibles fréquences $(<1 \mathrm{~Hz})$, la fréquence n'a pas un effet significatif sur le comportement cyclique des sols.

Les essais ont consisté à appliquer 700 cycles avec une contrainte déviatorique de $20 \mathrm{kPa}$ et une contrainte de confinement de $25 \mathrm{kPa}$. Dans le cas des échantillons qui n'ont pas atteint la rupture sous la sollicitation indiquée, le déviateur est progressivement augmenté par palier de $10 \mathrm{kPa}$ et, à chaque palier, 100 cycles ont été appliqués, ceci jusqu'à la rupture du sol.

\section{s.8}

\section{Fatigue sur une presse MTS à fréquence élevée}

Afin d'étudier le comportement des sols à une fréquence proche de celle résultant de la circulation des trains de grande vitesse d'une part, et d'étudier l'effet de la fréquence d'autre part, on a réalisé des essais de fatigue sur une presse MTS à trois fréquences différentes de 5, 10 et $20 \mathrm{~Hz}$ sur des échantillons à différentes teneurs en eau. Les essais ont été réalisés en appliquant des cycles de contrainte de 35 à $55 \mathrm{kPa}$. Ces contraintes ont été définies en considérant la contrainte générée par les structures d'assises ferroviaires, ainsi que la partie supérieure des terrassements (PST) traitée soit environ une contrainte verticale de $35 \mathrm{kPa}$. La charge cyclique de 0 à $20 \mathrm{kPa}$ induite par le passage d'un train à grande vitesse (TGV) retenue est issue des modélisations numériques effectuées par Géodynamique et Structures (vitesse particulaire sous la plate-forme ferroviaire de $30 \mathrm{~mm} / \mathrm{s}$, accélération de $0,6 \mathrm{~m} / \mathrm{s}^{2}$ et incréments de contraintes de $20 \mathrm{kPa}$ ). Les mesures effectuées ultérieurement sur le site de Beugnâtre (SEGG et SNCF) ont confirmé l'ordre de grandeur des sollicitations avec des accélérations verticales mesurées de $1 \mathrm{~m} / \mathrm{s}^{2}$.

L'échantillon a été préparé par carottage $(200 \mathrm{~mm}$ de diamètre et $30 \mathrm{~mm}$ de hauteur) et introduit ensuite dans une cellule odométrique. L'ensemble a été placé sur un support spécialement conçu pour cette étude. La figure 4 présente une photo du dispositif utilisé.

Dans un premier temps on applique une charge statique de $45 \mathrm{kPa}$. Après la stabilisation de la déformation, on applique la charge cyclique avec une amplitude de $10 \mathrm{kPa}$, ce qui correspond au chargement initialement défini de 35 à $55 \mathrm{kPa}$. 


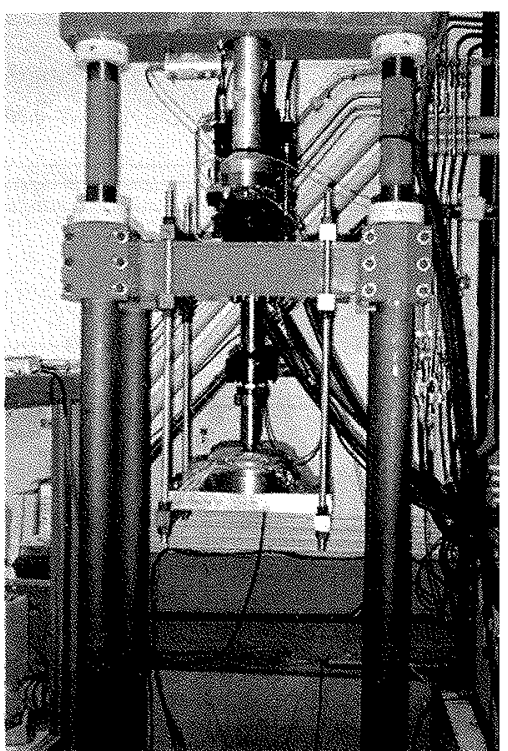

fc. 4 Dispositif utilisé pour l'essai de fatigue à haute fréquence.

Experimental set-up for fatigue tests at high frequency.
Pour un essai en chargement cyclique, on découpe un bloc de sol de $300 \times 150 \times 150 \mathrm{~mm}$; on le taille au tour manuel pour obtenir un échantillon cylindrique de $140 \mathrm{~mm}$ de hauteur et de $70 \mathrm{~mm}$ de diamètre; on procède à la saturation en réalisant la circulation de $\mathrm{CO}_{2}$ pendant $30 \mathrm{~min}$, la percolation de l'eau désaérée d'un volume supérieur à 2 volumes de pores (1,1 litre), et l'application d'une contre-pression par palier, jusqu'à atteindre les valeurs finales suivantes : $100 \mathrm{kPa}$ de contre-pression et $125 \mathrm{kPa}$ de pression de confinement; on applique le déviateur cyclique à une fréquence de $0,05 \mathrm{~Hz}$. Pour chaque sol, trois déviateurs différents sont considérés, correspondant respectivement à un tiers, deux tiers et la totalité du déviateur au pic obtenu avec l'essai triaxial non drainé en chargement monotone.

Pour les essais monotones et les essais cycliques, la déformation axiale $\left(\varepsilon_{1}\right)$, la surpression interstitielle $(\delta u)$, la contrainte déviatorique $\left(q=\sigma_{1}-\sigma_{3}\right)$ et la contrainte moyenne effective $\left(p^{\prime}=\sigma_{1}^{\prime} / 3+2 \sigma_{3}^{\prime} / 3\right)$ sont enregistrées par un système d'acquisition. On note que la contrainte déviatorique q est calculée en considérant la force imposée F et une section d'échantillon corrigée $S_{c^{\prime}}$ comme :

$$
\mathrm{S}_{c}=\mathrm{S}_{0} /\left(1-\varepsilon_{1}\right)
$$

où $\mathrm{S}_{0}$ est la section initiale de l'échantillon.

\section{4}

\section{Résultats et discussions}

\section{4.}

\section{Comportement d'effondrement des sols}

L'effondrabilité des quatre sols a été étudiée à l'cedomètre en utilisant les méthodes du simple et du double œdomètre. Les résultats sont présentés dans le tableau II. On observe que c'est le sol à 2,2 m de profondeur qui est le plus effondrable, puisque son effondrement atteint plus de $3 \%$. Ceci est principalement lié à sa plus grande porosité $(0,49$, voir tableau I). La comparaison entre les résultats obtenus par la méthode du simple œedomètre et la méthode du double œedomètre montre globalement une bonne correspondance sauf pour le sol à $3,5 \mathrm{~m}$ de profondeur avec $0,6 \%$ d'effondrement identifié par la méthode du simple odomètre contre 1,4\% par la méthode du double œdomètre.

Afin d'évaluer l'effet de la teneur en eau initiale sur l'effondrabilité des sols, on a amené les sols à différentes teneurs en eau. La figure 5 présente l'effondrement du sol à 2,2 $\mathrm{m}$ de profondeur en fonction de la teneur en eau initiale. On observe que l'effondrement augmente linéairement avec la diminution de la teneur en eau. De plus, une bonne correspondance est obtenue pour toutes les teneurs en eau entre les méthodes du simple et du double odomètre.
On cisaille ensuite l'éprouvette sur une presse triaxiale classique à une vitesse constante de $0,5 \% / \mathrm{min}$.

\section{taBksuu in Effondrement des sols à l'état naturel.} Collapse of soils from their natural states.

\begin{tabular}{|c|c|c|c|}
\hline Protordevi (m) & W (w) & Par siniple ceetometre $(\%$ ) & Pat dowbie didometre $(\%)$ \\
\hline 1,2 & 18,9 & 0,6 & 0,7 \\
\hline 2,2 & 18,1 & 3,2 & 3,7 \\
\hline 3,5 & 16,6 & 0,6 & 1,4 \\
\hline 4,9 & 23,7 & 0,2 & 0,4 \\
\hline
\end{tabular}




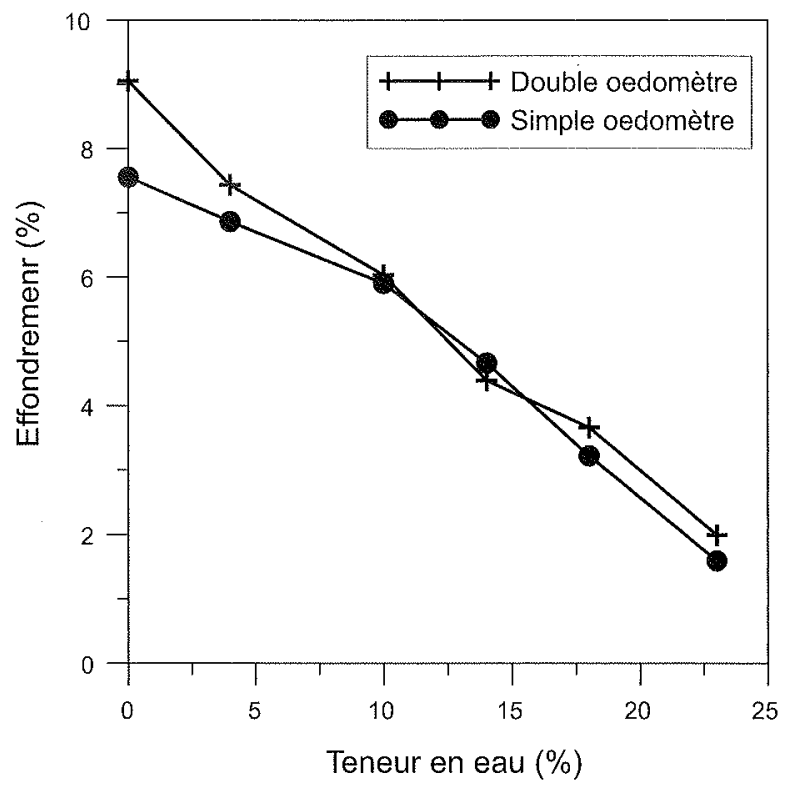

fic. 5 Effondrement du sol à $2,2 \mathrm{~m}$ à partir de différents états hydriques initiaux.

Collapse of soil at $2.2 \mathrm{~m}$ starting from different initial moisture states.

\section{Comportement en fatigue à faible fréquence}

Seul le sol à 2,2 m de profondeur a été testé au triaxial cyclique. Cinq teneurs en eau initiales différentes ont été considérées : 10, 14, 21,5, 23 et $27 \%$. Les essais ont commencé par l'application d'un déviateur de $20 \mathrm{kPa}$ pendant 700 cycles suivie de l'augmentation successive par paliers de $10 \mathrm{kPa}$ de déviateur avec 100 cycles pour chaque palier, jusqu'à la rupture du sol. Les résultats de la première phase ne sont pas présentés ici, qui montrent, d'une manière générale, une déformation assez limitée (inférieure à $0,5 \%$ ) pendant l'application du déviateur cyclique de $20 \mathrm{kPa}$. On observe que la déformation volumique et le déplacement vertical sont plus petits dans le cas des teneurs en eau faibles, ce qui est en bon accord avec l'augmentation de la rigidité du sol avec l'augmentation de la succion (Cui et Delage, 1996). Pour la teneur en eau de $10 \%$, la déformation est d'environ $0,15 \%$, tandis que pour des teneurs en eau de 21,5 et $23 \%$, proches de la limite de plasticité du sol, la déformation est autour de $0,5 \%$.

Les résultats de la deuxième phase de chargement sont regroupés sur les figures 6 et 7 , qui montrent respectivement les déformations axiale et volumique pour les différents états hydriques et pour les différents déviateurs cycliques. Pour la teneur en eau de $10 \%$, on observe que les déformations varient linéairement dans le temps, montrant un comportement volumique indépendant de l'amplitude du déviateur. Pour la teneur en eau de $14 \%$, on observe en revanche que l'évolution des déformations n'est plus linéaire : pour chaque palier de contrainte on a une déformation importante au départ qui tend à se stabiliser par la suite. De plus, les déformations augmentent avec l'augmentation de l'amplitude du déviateur. Le sol a atteint la rupture au dernier palier de charge, caractérisée par de grandes déformations. L'effet de l'amplitude semble s'accentuer avec l'augmentation de la teneur en eau. En effet, pour le même niveau de déviateur, les déformations sont d'autant plus importantes que la teneur en eau est élevée.

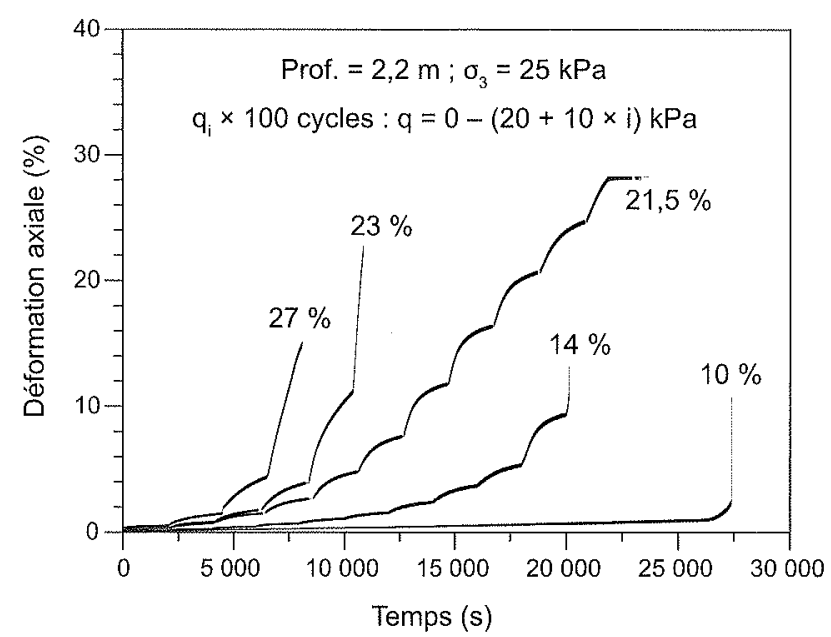

fic. 6 Évolution de la déformation axiale pour différents états hydriques et différentes amplitudes du déviateur cyclique; sol à $2,2 \mathrm{~m}$.

Evolution of axial strain for different moisture states and various amplitudes of cyclic deviator stresses; soil at $2.2 \mathrm{~m}$

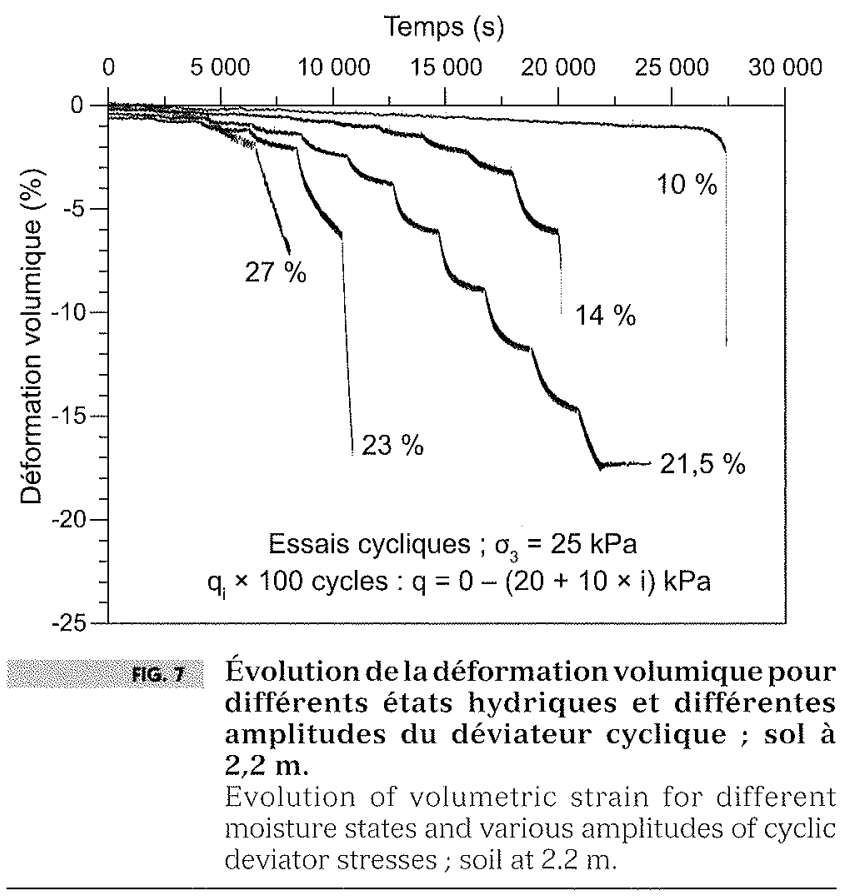

Vu que chaque palier correspond à $10 \mathrm{kPa}$ d'augmentation de déviateur cyclique, le nombre de paliers pour chaque teneur en eau représente la résistance au cisaillement du sol. On observe qu'à l'exception de l'essai à 21,5\% de teneur en eau, tous les essais montrent une diminution de la résistance au cisaillement avec l'augmentation de la teneur en eau. Ce phénomène est bien connu sur les sols non saturés sous sollicitations monotones (Escario et Saez, 1986 ; Delage et Graham, 1995 ; Drumright et Nelson, 1995, parmi d'autres) et a été pris en compte dans la loi de comportement de Barcelone (Alonson et al., 1990), connue sous le nom de BBM (Basic Barcelona Model) en termes d'effets de succion. 


\section{Comportement en fatigue à fréquence élevée}

La figure 8 présente les résultats de deux essais à deux teneurs en eau différentes (10 et $25 \%$ ) sur le sol à $3,5 \mathrm{~m}$ de profondeur. Chaque essai comporte les chargements de 35 à $55 \mathrm{kPa}$ à trois fréquences différentes : 5,10 et $20 \mathrm{~Hz}$. Pour chaque fréquence, environ 1 million de cycles ont été appliqués.

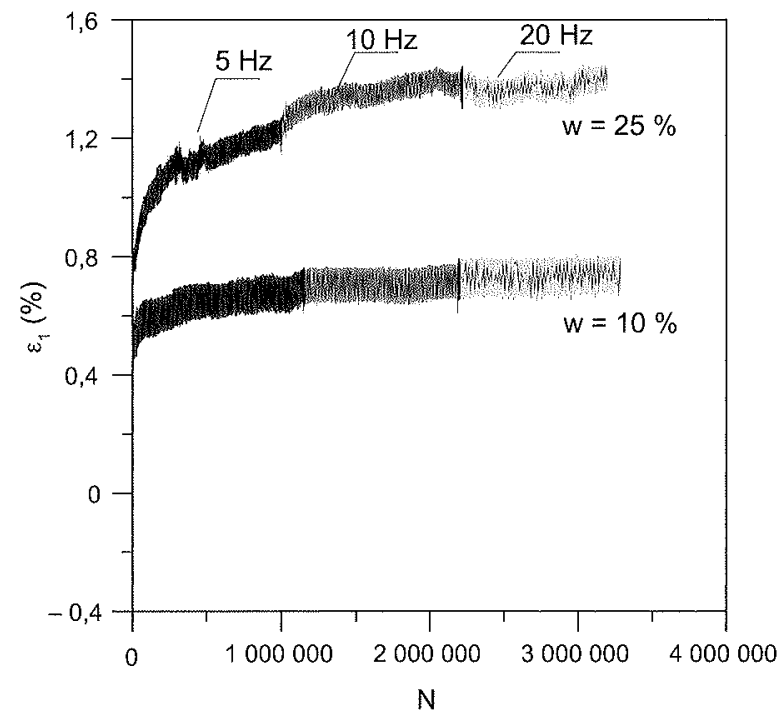

He. B Évolution de la déformation axiale à différents états hydriques et à différentes fréquences; sol à $3,5 \mathrm{~m}$.

Evolution of axial strain at different moisture states and frequencies; soil at $3.5 \mathrm{~m}$.
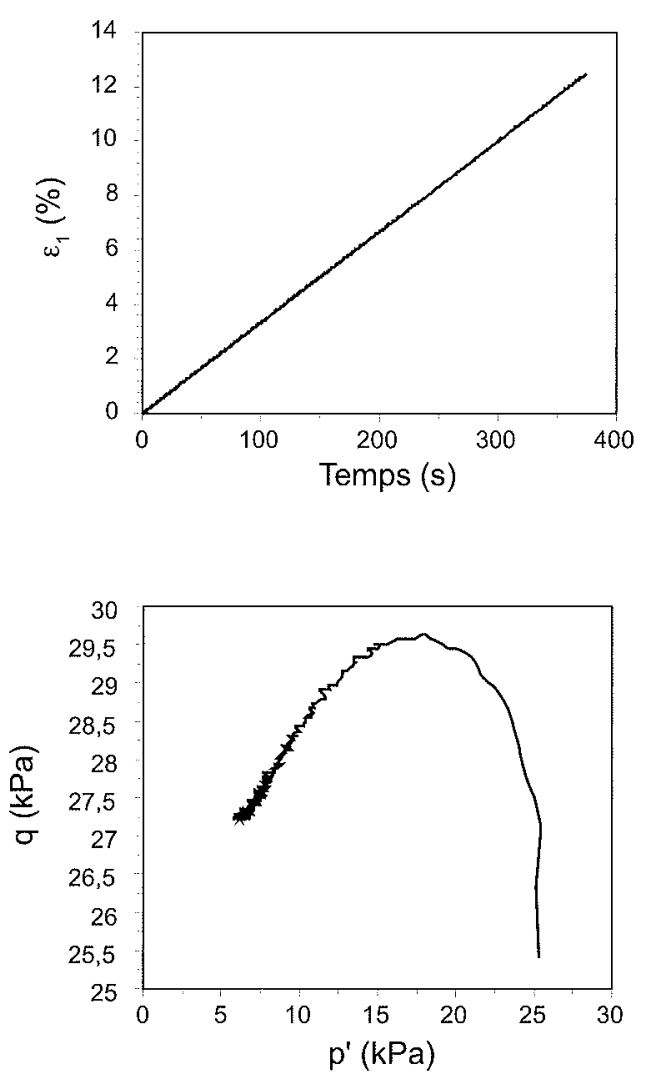

Pour la teneur en eau de $10 \%$, on observe que l'évolution de la déformation axiale est faible ; ceci quelle que soit la fréquence. Pour la teneur en eau de $25 \%$, on observe au départ à $5 \mathrm{~Hz}$ une évolution rapide, similaire à ce qu'on a observé au triaxial à faible fréquence pour un déviateur donné. Cette évolution s'atténue progressivement avec l'augmentation du nombre de cycles. Quand on passe à des fréquences plus élevées de 10 et $20 \mathrm{~Hz}$, la courbe semble se prolonger dans la continuité, montrant un effet négligeable de la fréquence.

\section{5}

\section{Comportement de liquéfaction}

La figure 9 présente les résultats de l'essai de cisaillement non drainé en chargement monotone sur le sol à 2,2 m de profondeur, avec l'évolution de la déformation axiale $\varepsilon_{1}$ en fonction du temps, la variation de la pression interstitielle $\delta u$ en fonction de $\varepsilon_{1}$, le chemin de contrainte dans le plan $\mathrm{p}^{\prime} / \mathrm{q}$ (contrainte moyenne effective/déviateur), et la variation du déviateur $q$ en fonction de $\varepsilon_{1}$.

On observe que le déviateur augmente rapidement au début du cisaillement jusqu'à $13,9 \mathrm{kPa}$, et qu'il diminue ensuite pour se stabiliser finalement sur une valeur de $6,8 \mathrm{kPa}$. Si on considère l'effondrement de la microstructure du sol comme étant l'origine du phénomène de liquéfaction, on peut conclure que pour cet essai la liquéfaction est amorcée quand le déviateur dépasse $13,9 \mathrm{kPa}$. Ce résultat est confirmé par la variation de la pression interstitielle : on observe que cette dernière peut augmenter jusqu'à $21,5 \mathrm{kPa}$, proche de la contrainte de consolidation ( $25 \mathrm{kPa}$ ).
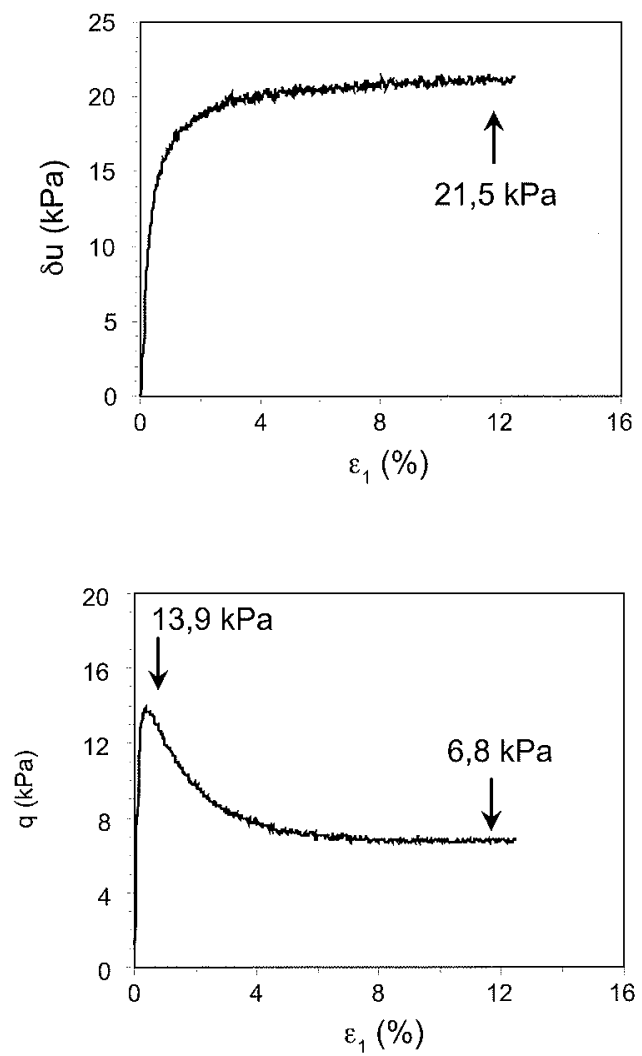

rG. 9 Essai de cisaillement non drainé en chargement monotone sur le sol à 2,2 m. Monotonic undrained shear test on soil at $2.2 \mathrm{~m}$. 
Cet essai en chargement monotone permet non seulement de mettre en évidence le caractère liquéfiable de ce sol, mais aussi de donner une valeur de déviateur limite pour des essais cycliques. Normalement, si le déviateur cyclique dépasse cette valeur limite $(13,9 \mathrm{kPa})$, le sol se liquéfierait en moins d'un cycle. Ainsi, on a défini les trois valeurs suivantes pour les essais cycliques : $8,4 \mathrm{kPa}, 11,7 \mathrm{kPa}$ et $14,9 \mathrm{kPa}$. On note que la dernière valeur dépasse légèrement le pic identifié

Le résultat de l'essai à $\mathrm{q}_{\mathrm{cyc}}=11,7 \mathrm{kPa}$ est présenté sur la figure 10. Une présentation quadratique composée des graphes $\varepsilon_{1} / t, \delta u / \varepsilon_{1}, p^{\prime} / q$ et $q / \varepsilon_{1}$ est adoptée. Sur le graphe $\varepsilon_{1} / t$, on observe que la déformation $\varepsilon_{i}$ évolue dans un premier temps de façon presque linéaire et avec une faible pente, et qu'elle évolue, en revanche, avec une forte pente après $800 \mathrm{~s}$ environ. La valeur de $\varepsilon_{\text {f }}$ correspondant au changement de taux est de $2 \%$ environ.

Sur le graphe $\delta u / \varepsilon$, on voit que la pression interstitielle augmente rapidement au début jusqu'à $19 \mathrm{kPa}$ environ (quand $\varepsilon_{1}$ est égal environ à $2 \%$ ). On atteint ensuite un plateau de stabilité avec une augmentation négligeable de pression interstitielle à chaque nouveau cycle de chargement. La valeur finale de la pression interstitielle est de $22 \mathrm{kPa}$ environ, proche de la contrainte de consolidation de $25 \mathrm{kPa}$. L'échantillon a donc atteint l'état de liquéfaction.

Sur le graphe $\mathrm{q} / \varepsilon_{1}$ on observe que pour des déformations axiales inférieures à $2 \%$, le déviateur reste presque constant $(11,7 \mathrm{kPa})$ et que les boucles effortdéformation sont serrées. En revanche, au-delà de $2 \%$ de déformation, le déviateur a tendance à décroître avec la déformation et les boucles devenant de plus en plus ouvertes. Cette diminution de contrainte déviatorique peut également être observée sur le graphe $p^{\prime} / q$, et est directement liée à la correction apportée qui est fonction de la force imposée et de la défor-
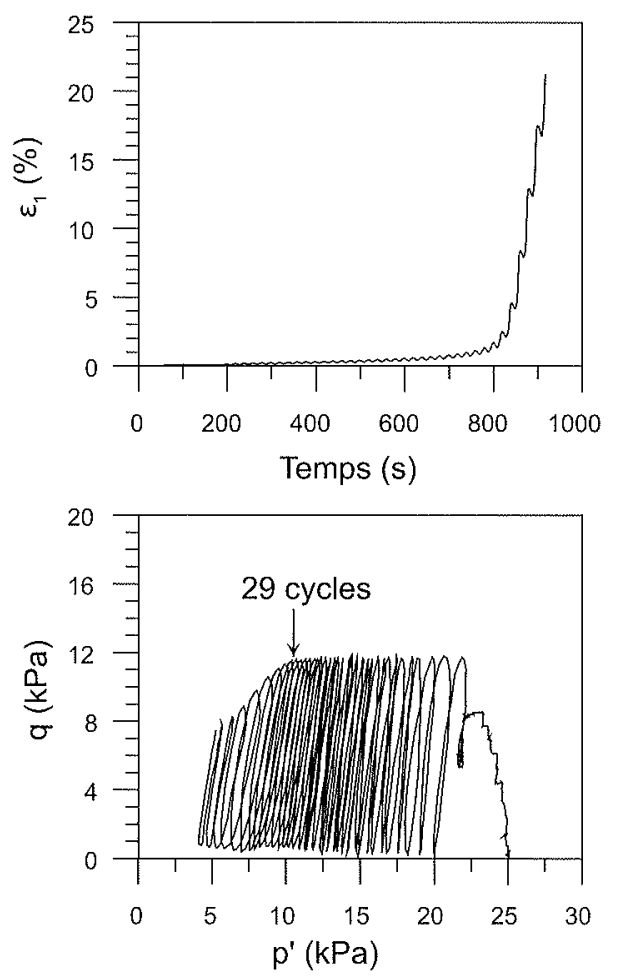

mation axiale (voir éq. 1). Il est intéressant de noter que, quand l'échantillon a atteint l'état de liquéfaction caractérisé par une surpression interstitielle proche de la contrainte de confinement à 29 cycles, une grande déformation s'amorce également. On peut ainsi définir pour cet essai un critère de liquéfaction soit en termes de surpression interstitielle soit en termes de déformation axiale.

La résistance à la liquéfaction en fonction du nombre de cycles pour le sol à 2,2 m est présentée sur la figure 11. On définit ici la résistance par la contrainte de cisaillement $\boldsymbol{\tau}_{\text {cyc }}=\frac{\sigma_{1}-\sigma_{3}}{2}=\frac{q_{\text {cyc }}}{2}$ normalisée par rapport à la contrainte de consolidation $\sigma^{\prime}$. On observe clairement que le nombre de cycles est d'autant plus grand que la contrainte de sollicitation est faible. Les trois autres sols ont été étudiés de la même manière et les résultats sont également présentés sur la figure 11. Pour le sol à $1,20 \mathrm{~m}$, les trois essais réalisés ont amené le sol à la liquéfaction; pour le sol à $3,50 \mathrm{~m}$, un seul essai a amené le sol à la licuéfaction; pour le sol à $4,90 \mathrm{~m}$, deux essais ont amené le sol à la liquéfaction. Si on compare la résistance à la liquéfaction des différents sols, on observe que c'est le sol à $1,20 \mathrm{~m}$ qui est le plus résistant et viennent ensuite le sol à $4,90 \mathrm{~m}$, le sol à $3,50 \mathrm{~m}$ et finalement le sol à 2,20 m.

Plusieurs travaux de recherche trouvés dans la littérature montrent que la résistance à la liquéfaction des sols sous chargements cycliques augmente avec l'augmentation de la densité (Seed et Idriss, 1971; Seed, 1979 ; Castro et Poulos, 1977 ; Vaid et al., 1985 ; Kramer et Seed, 1988; Alarcon-Guzman et al., 1988), de la fraction des particules fines (Ishihara, 1985 ; Kuerbis et al., 1988 ; Erten et Maher, 1995 ; Singh, 1994 et 1996), de l’indice de plasticité (Bray et Sancio, 2006), de la pression de consolidation (Casagrande, 1976 ; Tokimatsu et Hosaka, 1986; Yasuda et al., 1997), de l'effet du
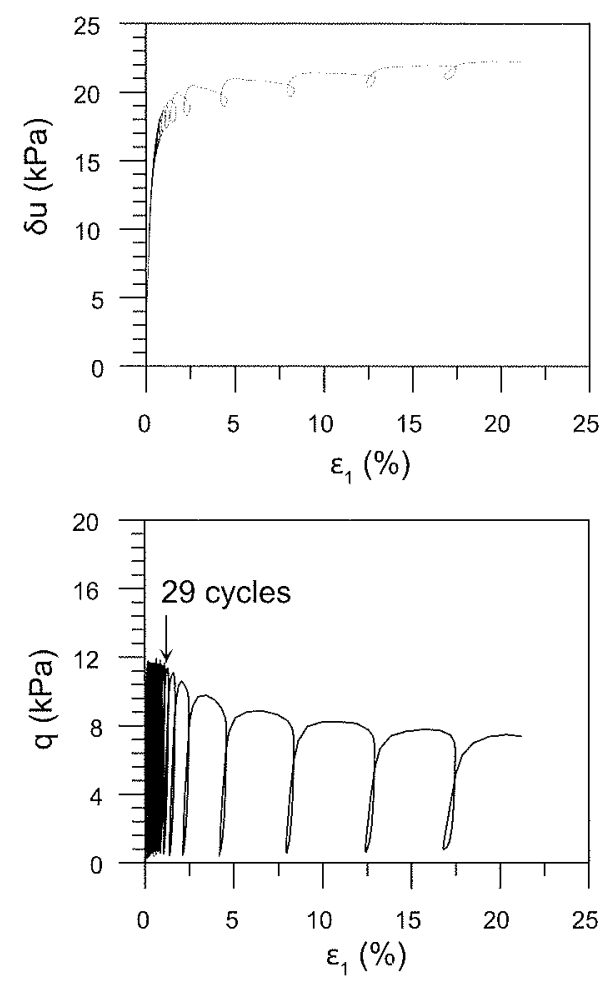


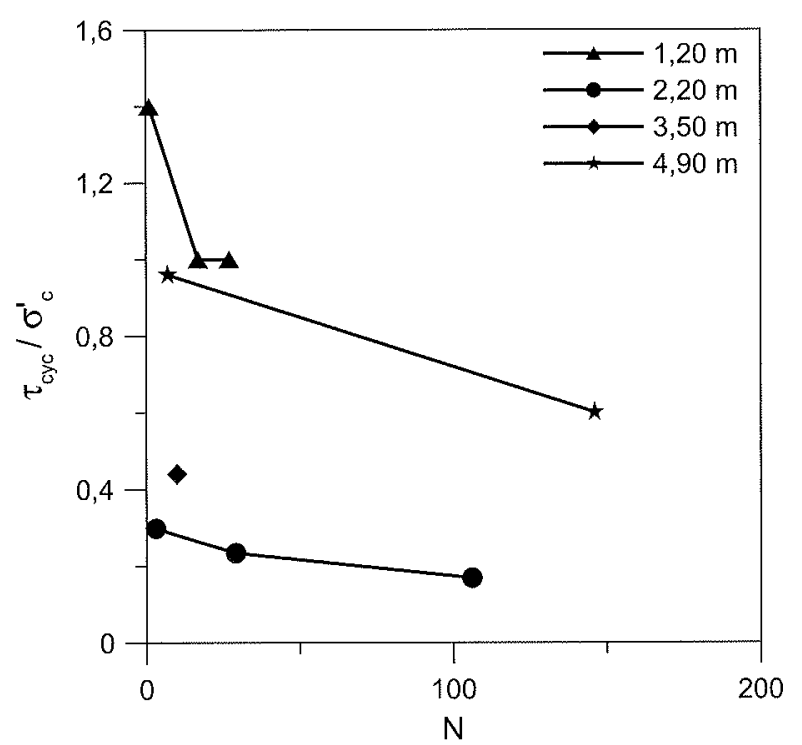

H6. 11 Courbes de résistance à la liquéfaction des quatre sols étudiés.

Curves of liquefaction resistance of the four soils studied.

vieillissement (Mitchell et Solymar, 1984 ; Skempton, 1986 ; Kulhawy et Mayne, 1990) et du degré de cimentation (Acar et El-Tahir, 1986 ; Dobry et al., 1982 ; Lade et Overton, 1989 ; Fernandez et Santamarina, 2001 ; Yun et Santamarina, 2005 ; Mohsin et Airey, 2005). Pour les quatre sols étudiés, comme le montre le tableau I, le sol à $2,2 \mathrm{~m}$ a la plus grande porosité $(n=0,49)$ et le sol à $1,2 \mathrm{~m}$ possède la fraction de fines la plus importante $(20 \%)$ et le plus grand indice de plasticité. Il est donc normal, conformément aux résultats trouvés dans la littérature, que le sol à 1,2 m soit le plus résistant et que le sol à 2,2 m soit le moins résistant à la liquéfaction.

\section{Conclusion}

Suite aux fontis observés sur la LGV Nord, une campagne d'essais a été réalisée en laboratoire visant à identifier les mécanismes d'instabilité liés au comportement du sol concerné : le loess. Les échantillons de sols intacts ont été prélevés en bloc à quatre profondeurs différentes et différents essais ont été réalisés, tels que l'essai d'identification géotechnique, l'essai d'effondrement à l'œedomètre, l'essai de fatigue à faible et grande fréquences, l'essai de liquéfaction.

Les essais d'identification ont montré que les sols étudiés sont caractérisés par un faible indice de plasticité (6-9), une faible densité sèche $\left(1,39-1,55 \mathrm{Mg} / \mathrm{m}^{3}\right)$ qui correspond à une grande porosité $(0,43-0,49)$ ou un grand indice des vides $(0,76-0,93)$ et une teneur en carbonate assez élevée (5-15\%). Ceci est caractéristique des sols lœssiques.
C'est le sol à 2,2 m qui s'est avéré le plus effondrable, à relier principalement à sa plus grande porosité. L'effet de la teneur en eau sur l'effondrabilité est important puisqu'on a observé une augmentation linéaire de l'effondrement avec la diminution de la teneur en eau. De plus, une bonne correspondance générale a été obtenue entre les méthodes du simple et du double oedomètre.

Les résultats des essais de fatigue à faible fréquence ont montré que les déformations augmentent généralement avec l'augmentation de l'amplitude du déviateur. Pour le même niveau de déviateur, les déformations sont d'autant plus importantes que la teneur en eau est élevée, montrant un effet croissant de l'amplitude au fur et à mesure de l'augmentation de la teneur en eau. Les essais réalisés à frécuence élevée ont montré un effet de fréquence négligeable.

Les essais de cisaillement non drainé sous chargement monotone ont montré le caractère liquéfiable des sols, avec une surpression interstitielle finale proche de la contrainte de consolidation. Les essais cycliques non drainés ont confirmé cette propriété de liquéfaction. De plus, les résultats ont montré qu'on peut définir un critère de liquéfaction soit en termes de surpression interstitielle soit en termes de déformation axiale. Le sol à 1,2 m s'est avéré le plus résistant et le sol à 2,2 m le moins résistant à la liquéfaction, ce qui est conforme aux résultats trouvés dans la littérature quant aux effets de la densité et de la fraction des fines.

Sur le plan pratique, cette étude de laboratoire a permis de répondre aux objectifs de caractérisation des phénomènes rencontrés (effondrement, fatigue et liquéfaction), mais également de les hiérarchiser du point de vue de l'exploitation ferroviaire en identifiant la liquéfaction comme un phénomène potentiellement critique.

Suite à ces résultats et à l'identification de la forte variation de comportement des lœess en fonction de leur état hydrique, la direction de l'ingénierie de la SNCF et RFF ont poursuivi ces recherches en évaluant cette sensibilité in situ. Cette partie d'étude non décrite dans le présent article, a permis de confirmer cette sensibilité de réponse, plus ou moins discriminante en fonction de l'essai retenu.

Une autre partie d'étude a ensuite été lancée visant à qualifier la sensibilité à la liquéfaction des loess en exploitant les résultats d'une campagne de reconnaissances classique, sans essais de laboratoire sophistiqués. Des développements spécifiques originaux ont dû être réalisés pour ramener les sols à l'état saturé à partir de résultats d'investigations in situ en conditions non saturées.

\footnotetext{
REMERCIOMENTS

Ce travail est dédié à Gilles Marchadier qui disparut soudainement le 29 mai 2007 à l'âge de 30 ans. Les auteurs adressent leur gratitude à RFF
} pour son support financier. 


\section{Bibliographie}

Acar Y.B., El-Tahir A. - Low Strain Dynamic Properties of Artificially Cemented Sand. Journal of Geotechnical Engineering. 112 (11), 1986, p. 1001-1015.

AFNOR - NF XP 94-202 - Géotechnique, Essais in situ, 1994, tome 2.

Alarcon-Guzman A., Leonards G.A., Chameau J.-L. - Undrained monotonic and cyclic strength of sands. Journal of Geotechnical Engineering, ASCE, 114 (10), 1988, p. 1089-1109.

Alonso E.E., Gens A., Josa A. - A constitutive model for partially saturated soils. Géotechnique, vol. 40 (3), 1990, p. 405430.

Bray J., Sancio R. - Assessment of the liquefaction susceptibility of fine-grained soils. Journal of Geotechnical and Geoenvironmental Engineering, 132 (9), 2006, p. 1165 1177.

Canou J., Benahmed N., Dupla J.-C., De Gennaro V. - Instabilité de liquéfaction et phénomène de mobilité cyclique dans les sables. Revue française de géotechnique, 98,2002 , p. 29-46.

Casagrande A. - Liquefaction and cyclic mobility of sands - a critical review. Harvard Soil Mechanics Series, $\mathrm{n}^{\circ} 88$, Harvard University, Cambridge, Mass, 1976.

Castro G., Poulos S.J. - Factors affecting licfuefaction and cyclic mobility. Journal of Geotechnical Engineering Division, ASCE, 103, 1977, p. 501-516.

Cui Y.-J., Delage P., Durand F., Schlosser F., Wojnarowicz M. - Comportement mécanique des loess sur le tracé du TGV Nord. Proceedings of the 11th ECSMFE, Copenhague, vol. 7, 1995, 45-50.

Cui Y.-J., Delage P. - Yielding and plastic behaviour of an unsaturated compacted silt. Géotechnique, 46 (2), 1996, p. 291 311.

Cui Y.-J., Delage P., Alzoghbi P. - Microstructure and collapse behaviour of a loess from northern France. Proc. of Unsat'98, Beijing, vol. 1, 1998, p. 31-36

Cui Y.-J., Magnan J.-P. - Affaissements locaux dus à l'infiltration d'eau (chap. 6). Géomécanique environnementale, risques naturels et patrimoine. Édition Hermès, 2000, p. 139-164.

Cui Y.-J., Tang A.M., Marcial D., Terpereau J.-M., Marchadier G., Boulay X - Use of a differential pressure transducer for the monitoring of soil volume change in cyclic triaxial test on unsaturated soils. Journal of Geotechnical Testing, 30 (3), 2007, p. 227-233.

Delage P., Graham J. - General report Mechanical behaviour of unsaturated soils : understanding the behaviour of unsaturated soils requires reliable conceptual models. Proc. of the First Conference on Unsaturated Soils, Unsat'95, Paris, vol. 3, 1995, p. 1223-1256.

Delage P., Cui Y.-J. - Leau dans les sols non saturés. Technique de l'ingénieur, C301 2000.

Dobry R., Ladd R.S., Yokel F.Y., Chung R.M., Powell D. - Prediction of pore water pressure buildup and liquefaction of sands during earthculakes by the cyclic strain method. NBS Building Science Series 138, National Bureau of Standards, Gaithersburg, MD, 1982, $150 \mathrm{p}$

Drumright E.E., Nelson J.D. - The shear strength of unsaturated tailings sand. Proc. of the First Conference on Unsaturated Soils, Unsat'95, Paris, vol. 1, 1995, p. $45-50$.

Erten D., Maher M.H. - Liquefaction potential of silty soils. Soil Dynamic and Earthquake Engineering. Proc. VII Int Conf., A.S. Cakmak and C.A. Brebbia, eds., Elsevier Science, London, 1995 p. 163-171.

Escario V., Saez J. - The shear strength of partly saturated soils. Géotechnicue, 36 (3). 1986, p. 453-456.

Fernandez A.L., Santamarina J.C. - Effec of cementation on the small-strain parameters of sands. Can. Geotech. J. 38 (1), 2001, p. 191-199

Ishihara K. - Stability of natural deposits cluring earthquakes. Proceed. 11th International Conference on Soil Mechanics and Foundation Engineering. Balkema, Rotterdam, vol. 1, 1985, p. 321-376.

Jennings J.E., Knight K. - The additional settlement of foundation due to collapse of sandy soils on wetting. Proc. 4th ICS MFE, vol. 1, 1957, p. 316-319.

Knight K. - The origin and occurrence of collapsing soils. Proc. 3rd Reg. African CSMFE, vol. 1, 1963, p. 127-130.

Kramer S.L., Seed H.B. - Initiation of soi liquefaction under static loading conditions. Joumal of Geotechnical Engineering, ASCE, 114, 1988, p. 412-430.

Kuerbis R.H. Negussey D., Vaid Y.P. - Effect of gradation and fines content on the undrained response of sand. Geotechnical Special Publication, $n^{\circ}$ 21. ASCE New York, 1988, p. 330-345

Kulhawy F.H., Mayne P.W. - Manual on estimating soil properties for foundation design. Final report 1493-6, EL-6800 Electric Power Research Institute, Palo Alto, Calif., 1990, 306 p.

Lade P.V., Overton D.D. - Cementation effects in frictional materials. Journal of Geotechnical Engineering, 115 (10), 1989, p. 1373-1387.
Mitchell J.K., Solymar Z.V - Time-Dependent Strength Gain in Freshly Deposited or Densified Sand. J. Geotech. Eng. ASCE, 110 (11), 1984, p. 1559-1576.

Mohsin A.K.M., Airey D.W. - Influence of cementation and density on $G$ for sand. Proceedings of the 16th International Conference on Soil Mechanics and Geotechnical Engineering, 2, 2005 p. 413-416

Osipov V.I., Sokoloy V.N. - Factors and mechanism of loess collapsibility. Genesis and Properties of Collapsible Soils, E. Derbyshire et al. eds, NATO - ASI Series, Kluwer Ac. Publ., 1995, p. 49-63.

Skempton A.W. - 1 - Standard penetration test procedures and the effects of overburden pressure, relative density, particle size, ageing, and overconsolidation. Geotechnique, 36 (3), 1986, p. 425-447.

Seed H.B., Idriss I.M. - Simplified procedure for evaluating soil liquefaction potential. Journal of Soil Mechanics and Foundation Division, ASCE, 97 (SM9) 1971 , p. 1249-1273.

Seed H.B. - Soil liquefaction and cyclic mobility evaluation for level ground during earthquakes. Joumal of GeotechniCal Engineering Division, ASCE, 105 (2), 1979, p. 201-255.

Singh S. - Re-examination of the effects of fine contents on the liquefaction characteristics of sands. Earthquake Geotechnical engineering, $\mathrm{K}$. Ishihara, ed., Balkema, Rotterdam, The Netherlands, 1994, p. 829-831.

Singh S. - Liquefaction characteristics of silts. Geotechnical and Geological Engineering, 14 (1), 1996, p. 1-19.

Tokimatsu K., Hosaka Y. - Effects of sample disturbance on dynamic properties of sand. Soils Foundations, 26 (1), 1986, p. 53-64.

Vaid Y.P., Chern J.C. - Cyclic and monotonic undrained response of saturated sands. Advances in the art of testing soils under cyclic conditions. V. Khosla ed., American Society of Civil Engineers (ASCE), New York, 1985, p. 120-147.

Yasuda S., Ishihara K., Harada K. Nomura H. - Factors which affected the area of lateral flow that occurred in the ground behind quaywalls. Proc. 2nd Conf. on Earthquake disasters in Hanshin-Awaii area, JSCE, 1997, p. 113-120 (in Japanese)

Yun S.T., Santamarina J.C. - Decementation, softening, and collapse : changes in small-strain shear stiffness in $\mathrm{k} 0$ loading. Journal of Geotechnical and Geoenvironmental engineering, 131 (3), 2005, p. 350-358. 